\title{
Rapid propagation of a Bloch wave packet excited by a femtosecond ultraviolet pulse
}

\author{
E. E. Krasovskii, ${ }^{1,2,3}$ C. Friedrich, ${ }^{4}$ W. Schattke, ${ }^{2,5}$ and P. M. Echenique ${ }^{1,2,6}$ \\ ${ }^{1}$ Departamento de Física de Materiales, Universidad del Pais Vasco UPV/EHU, San Sebastián/Donostia, Spain \\ ${ }^{2}$ Donostia International Physics Center (DIPC), Paseo Manuel de Lardizabal 4, San Sebastián/Donostia, Spain \\ ${ }^{3}$ IKERBASQUE, Basque Foundation for Science, 48013 Bilbao, Spain \\ ${ }^{4}$ Peter Grünberg Institut and Institute for Advanced Simulation, Forschungszentrum Jülich and JARA, 52425 Jülich, Germany \\ ${ }^{5}$ Institut für Theoretische Physik und Astrophysik der Christian-Albrechts-Universität zu Kiel, 24118 Kiel, Germany \\ ${ }^{6}$ Centro de Física de Materiales CFM - Materials Physics Center MPC, Centro Mixto CSIC-UPV/EHU, 20018 San Sebastián/Donostia, Spain
}

(Received 18 May 2016; revised manuscript received 4 July 2016; published 22 November 2016)

\begin{abstract}
Attosecond streaking spectroscopy of solids provides direct observation of the dynamics of electron excitation and transport through the surface. We demonstrate the crucial role of the exciting field in electron propagation and establish that the lattice scattering of the outgoing electron during the optical pumping leads to the wave packet moving faster than with the group velocity and faster than the free electron. We solve the time-dependent Schrödinger equation for a model of laser-assisted photoemission, with inelastic scattering treated as electron absorption and alternatively by means of random collisions. For a weak lattice scattering, the phenomenological result that the photoelectron moves with the group velocity $d E / d \hbar k$ and traverses on average the distance equal to the mean-free path is proved to hold even at very short traveling times. This offers a novel interpretation of the delay time in streaking experiment and sheds new light on tunneling in optoelectronic devices.
\end{abstract}

DOI: 10.1103/PhysRevB.94.195434

Transport properties of electron wave packets underlie the functioning of electronic devices and are an important factor in photoemission spectroscopies and electron diffraction techniques [1]. Still, little is known about how a photoexcited Bloch wave packet emerges out of the initial state and develops on a time scale comparable to its lifetime. Such ultrafast excitations are probed by time-resolved photoelectron spectroscopies, such as interference of two-photon transitions [2] or laser streaking [3-10]. In the latter technique, a subfemtosecond time resolution is achieved by mapping time to energy using a strong laser field. The electron wave packet created by a femtosecond pulse of extreme ultraviolet radiation (XUV) is accelerated by the superimposed laser field, and the energy by which its spectrum shifts up or down indicates the electron release time $t_{\mathrm{X}}$ relative to the temporal profile of the laser field $\mathcal{E}_{\mathrm{L}}(t)$. In many cases the low-frequency laser field is strongly damped by the dielectric response [11], so the photoelectron needs to travel some distance before it gets exposed to the streaking field. This is the basis of a number of theories proposed to describe the electron dynamics in the attosecond experiment [12-16].

The key questions are how much time does the excited electron spend in the solid before it escapes into vacuum and what is its velocity? Here, we discover the crucial role the exciting light pulse plays in the electron dynamics in a streaking experiment: the Bloch wave packet excited close to a gap in the energy spectrum moves faster than with the group velocity. The reason is that its spectrum keeps evolving while it moves. The size of the effect depends on inelastic scattering, so to validate the relevance of our calculations to the actual photoemission process we numerically establish the equivalence of the results obtained with an absorbing potential and with a statistical averaging over random perturbations. Further, we verify the accuracy of the laser-streaking clock by comparing it with the exact clock based on solely the spatial motion of the packet.
Once the wave packet is created, its time of flight to the surface is determined by its group velocity $v$. Owing to inelastic scattering, the photoelectron excited at a depth $z$ has the probability $\exp (-z / \lambda)$ to reach the detector, so the average traversed depth is just the mean-free path (MFP) $\lambda$, and the time to reach the surface is $\tau=\lambda / v$ [8]. Inelastic scattering enters the Hamiltonian through the imaginary optical potential $-i V_{\mathrm{i}}$. For nearly free electrons $\lambda$ is proportional to $v$, so that $\tau$ depends on energy solely through $V_{\mathrm{i}}(E)$, Supplemental Material A [17]:

$$
\tau=\frac{\hbar}{2 V_{\mathrm{i}}} .
$$

The optical potential changes smoothly with energy and is similar for different materials, as suggested by experience with angle-resolved photoemission (ARPES) [18,19,23] and very low-energy electron diffraction (VLEED) [20,21,24], Fig. 1(c). Thus, $\tau$ is expected to change slowly with energy, irrespective of MFP, which may rapidly vary following the group velocity, Fig. 1(b) [25]. Microscopically, the optical potential is associated with the imaginary part of the self-energy [23]. Figure 1(c) includes corresponding ab initio results for a high-lying free-electron-like conduction band of W along $\Gamma N$ obtained within the $G W$ approximation [26]. The good agreement of the calculated $V_{\mathrm{i}}(E)$ with the empirical results supports our understanding of the electron absorption as coming predominantly from the electron-electron interaction and confirms the empirical estimate of its growth with energy.

Thus, the higher the electron energy the earlier it should escape, see Fig. 1(d). The measurements on W(110) [22] of the time delay between $5 d$ valence band (VB) and semicore $4 f$ states qualitatively follow this trend [Fig. 1(d)], but experimental values are about three times larger. On the contrary, the value of $5 \pm 20$ asec for the delay of core $\mathrm{Mg} 2 p$ electrons relative to $\mathrm{VB}$ measured on $\mathrm{Mg}(0001)$ at $\hbar \omega=118 \mathrm{eV}$ is much smaller than follows from Eq. (1). Below, we present a mechanism that may be responsible for 

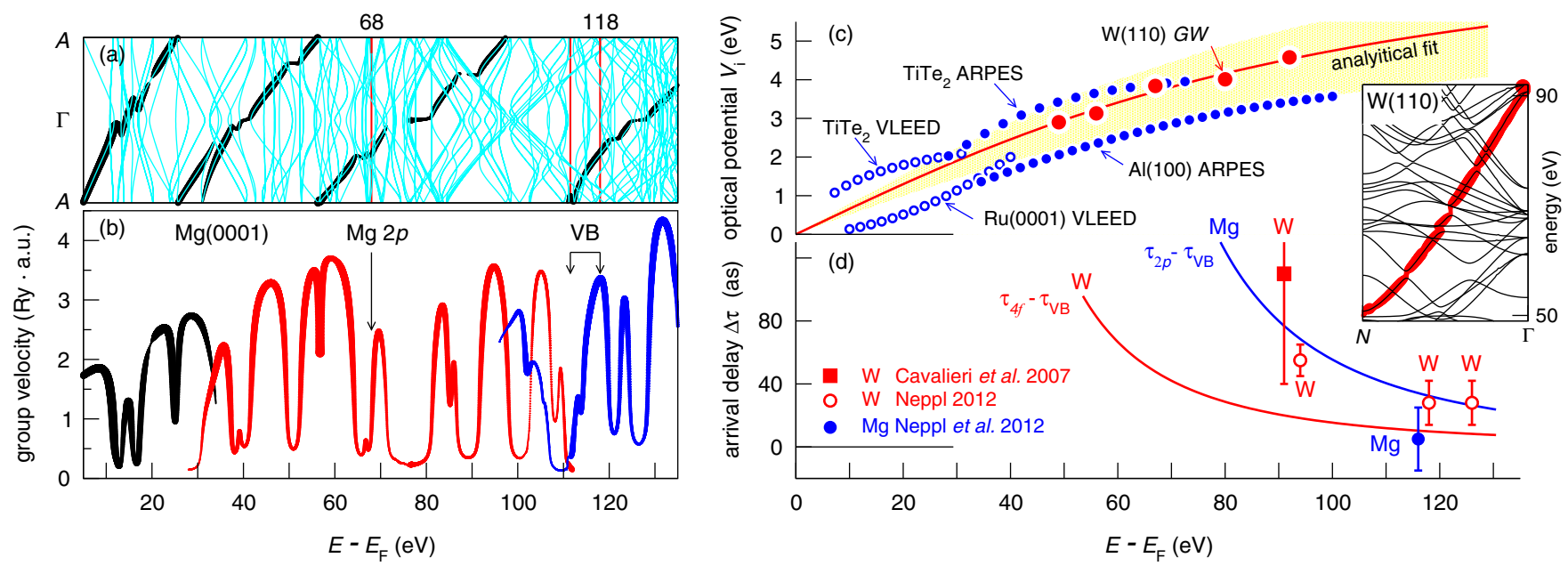

FIG. 1. (a) Real band structure of $\operatorname{Mg}(0001)$ (thin lines) and dispersion $E(k)$ of conducting Bloch waves (see also Supplemental Material B [17]): line thickness indicates the current carried by the wave as a constituent of the outgoing beam. Only the fragments related to real $k$ are shown. (b) Velocity of conducting waves (including complex band structure). Arrows show the final states for emission from Mg $2 p$ and from VB. (c) $V_{\mathrm{i}}(E)$ empirically derived from ARPES of $\mathrm{TiTe}_{2}$ [18] and $\mathrm{Al}(100)$ [19] (small full circles) and from VLEED of TiTe 2 [20] and $\mathrm{Ru}(0001)$ [21] (open circles). Large circles are the ab initio $G W$ calculation for W(110) for the band highlighted in the inset. (d) Arrival delay as a function of the final-state energy according to Eq. (1). Top (blue) curve is for the emission from $\mathrm{Mg}(0001): \mathrm{Mg} 2 p$ band relative to $\mathrm{VB}$ $\left(E_{\mathrm{VB}}-E_{2 p}=50 \mathrm{eV}\right)$, and bottom (red) curve is for $\mathrm{W}(110)$ : W $4 f$ band relative to $\mathrm{VB}\left(E_{\mathrm{VB}}-E_{4 f}=28 \mathrm{eV}\right)$. For W(110), the function $V_{\mathrm{i}}(E)$ is the analytical fit to $G W$ values [graph (c)], and for $\operatorname{Mg}(0001)$ we used a fit to the $\mathrm{Al}(100)$ data. The red square and open red circles are experimental results for W(110) of Ref. [8] and of Ref. [22], respectively. Full blue circle is the measurement of Ref. [9] for Mg.

such drastic disagreement and point to the cases in which Eq. (1) does not hold.

For the proof-of-concept calculation we employ a numerically exact particle-in-the-box model that makes no assumptions about the electron dynamics in a periodic potential and does not separate excitation from transport. The box comprises a thick one-dimensional (1D) crystal slab on a structureless substrate [potential $V(z)$ in Fig. 2(a)] and the vacuum half space, see Supplemental Material C [17] and Ref. [27].

We perform a series of numerical experiments, in which electrons are excited by an XUV pulse of duration $D_{\mathrm{X}}=1$ fs and are simultaneously acted upon by the laser pulse $\mathcal{E}_{\mathrm{L}}(t)$ of duration $5 \mathrm{fs}$, photon energy $1.65 \mathrm{eV}$ and amplitude $\mathcal{E}_{\mathrm{L}}^{\mathrm{M}}=2 \times 10^{7} \mathrm{~V} / \mathrm{cm}$, see Fig. 2(b) and Supplemental Material $\mathrm{D}$ [17]. The laser field is screened by multiplying by a smooth step function $\theta(z)$. The time-dependent Schrödinger equation is solved in matrix form in terms of exact eigenfunctions of the unperturbed Hamiltonian $\hat{H}=\hat{p}^{2} / 2 m+V$, so the crystal potential is fully taken into account for both initial and final states [27,28]. Apart from $\hat{H}$ and the two external fields the Hamiltonian includes inelastic scattering in two alternative ways: it is either a static absorbing potential $-i V_{\mathrm{i}}[1-\theta(z)]$ or a real stochastic potential $\sigma_{n}(t, z)[1-\theta(z)]$ followed by averaging over random configurations $\sigma_{n}$, Supplemental Material E [17].

The displacement $\Delta E$ of the spectrum from its laser-free position [Fig. 2(e)] as a function of the time shift $\Delta t=t_{\mathrm{L}}-t_{\mathrm{X}}$ between the XUV and the laser pulse provides the temporal information: by fitting the measured $\Delta E(\Delta t)$ points with the momentum transfer function $p(\tau)=e \int_{\tau}^{\infty} d t \mathcal{E}_{\mathrm{L}}\left(t-t_{\mathrm{X}}\right)$, we infer the time $\tau$ at which the electron appears in vacuum, see the shift of the curves in Figs. 2(c) and 2(d).
First, we consider a setup where the photoelectron initial position is known: we introduce a small defect at one of the layers [Fig. 2(a)] and photoexcite the localized state at the defect. Figures 2(c) and 2(d) show streaking curves for the initial state at the third layer for XUV photon energies $\hbar \omega=78$ and $90 \mathrm{eV}$. Counterintuitively, the electron at the higher energy arrives 40 asec later than at the lower energy: $\tau=150 \mathrm{asec}$ at $90 \mathrm{eV}$ and $\tau=111 \mathrm{asec}$ at $78 \mathrm{eV}$. Note that phenomenological absorbing potential and microscopic random collisions agree both in the temporal shift of the streaking curves, Figs. 2(c) and 2(d), and in the energy shift of the individual spectra, Fig. 2(e). Figure 2(g) shows the escape time of the electron initially at the second, third, and fourth layer for $\hbar \omega=65$ to $159 \mathrm{eV}$ (initial state energy is $\left.E_{\text {ini }}=-41.2 \mathrm{eV}\right)$. The most striking are the two minima at $E=\hbar \omega+E_{\text {ini }}=39$ and at $102 \mathrm{eV}$ [ $A$ and $C$ in Fig. 2(g)], at which $\tau$ even shows negative values [29].

The minima $B$ and $C$ are located at the lower edges of the band gaps, and minimum $A$ is due to the vanishing ionization cross section (Cooper minimum), see Fig. 2(f). There $\tau(E)$ strongly deviates from the function $d / v(E)$ with $v(E)=d E / d \hbar k$ [dashed curve in Fig. 2(g)]. To prove that the discrepancy does not arise from the rather indirect streaking method to measure $\tau$, we use an alternative clock: we switch off the laser and measure the wave packet's equation of motion in vacuum $z_{0}+\tilde{v} t$ [inset of Fig. 2(i)] to obtain the time point $t_{0}$ at which it has crossed the surface. The curves $t_{0}(E)$ and $\tau(E)$ agree well, Fig. 2(h) and 2(i), so the laser-streaking clock is reliable.

The fast delivery of the photoelectron to the surface is due to the pump excitation: Fig. 3 shows the time evolution of the spectrum at the $\tau(E)$ minimum, $\hbar \omega=80 \mathrm{eV}$, and at $\hbar \omega=100 \mathrm{eV}$, where $\tau(E)$ well agrees with the instantaneous 

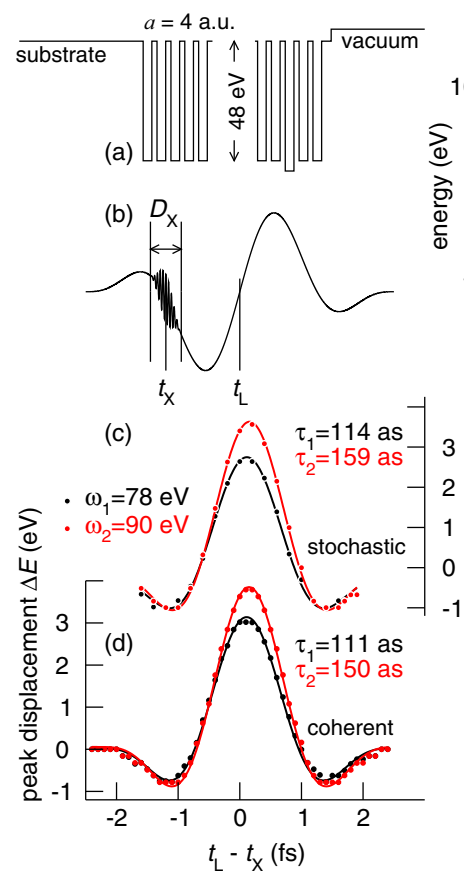
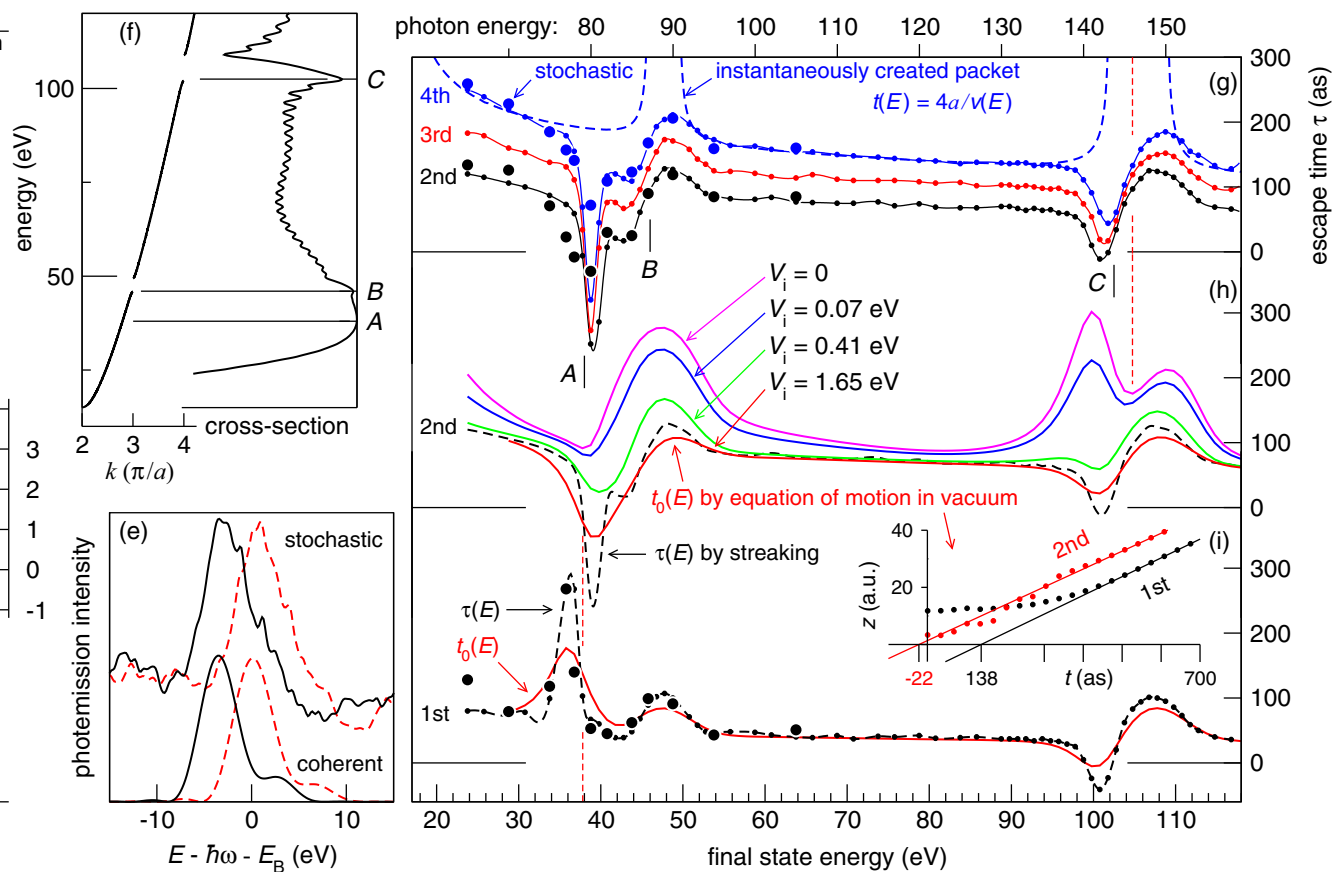

FIG. 2. Escape time from the streaking clock for a localized state. (a) Crystal potential $V(z)$ with a defect at the third layer. (b) Superposition of the XUV and laser pulses. (c) Streaking curves for $\hbar \omega=78$ and $90 \mathrm{eV}$ with random collisions; (d) the same with absorbing potential. (e) Streaked spectra for $\hbar \omega=78 \mathrm{eV}$ with absorption (lower curves) and with random collisions (top curves) for $t_{\mathrm{L}}-t_{\mathrm{X}}=1600$ asec (solid lines) and 200 asec (dashed lines). (f) Band structure with the periodic potential $V(z)$ and energy dependence of the emission intensity from a localized state at a defect. (g)-(i) Escape time as function of the final energy $E=\hbar \omega-41.2 \mathrm{eV}$. Solid lines connecting small circles in (g) and dashed lines in (h) and (i) are streaking results with absorption. Large circles in (g) and (i) are streaking with random collisions. In (g), initial state is at the second (black), third (red), and fourth (blue) layer; dashed curve is the depth divided by the group velocity. In (h) and (i), solid lines are from the equation of motion $z(t)$ in vacuum for initial state in the second (h) and in the first (i) layer, for $V_{\mathrm{i}}=0,0.07,0.41$, and $1.65 \mathrm{eV}$. Inset shows $z(t)$ for initial state in the first (black) and second (red) layer. The relevant final energy is indicated by the red dashed vertical bar in (i).

approximation $d / v(E)$. The two spectra evolve qualitatively differently: while at $100 \mathrm{eV}$ the intensity rapidly concentrates around $E=\hbar \omega+E_{\mathrm{ini}}$, at $80 \mathrm{eV}$ the evolution is much slower: at $t=200$ asec the spectrum is still spread over a range of $40 \mathrm{eV}$. Clearly, while the XUV pulse is on and the spectral coefficients $\psi(E, t)$ of the packet $\int d E \psi(E, t)|E\rangle$ keep changing, the centroid velocity generally deviates from the
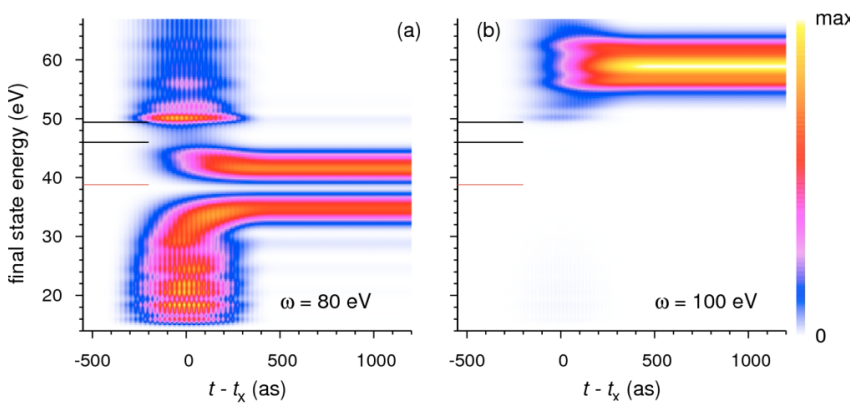

FIG. 3. Temporal evolution of the photoelectron spectrum from a localized state. XUV pulse duration is $D_{\mathrm{X}}=1000$ asec. (a) XUV photon energy $\hbar \omega=80 \mathrm{eV}$. (b) $\hbar \omega=100 \mathrm{eV}$. The black bars at 46 and $50 \mathrm{eV}$ indicate a spectral gap, and the red bar at $39 \mathrm{eV}$ indicates the Cooper minimum, see Fig. 2(f). For the sake of better clarity, the maps are presented for $V_{\mathrm{i}}=0$. weighted group velocity of the states $|E\rangle$. Such unusual behavior happens every time when the central energy of the wave packet approaches an intensity minimum, be it a vanishing matrix element or a spectral gap. Note that for the emission from the first layer there is a retardation by about 200 asec, which is apparently caused by the asymmetry of the excitation cross section at the surface.

The comparison in Fig. 2(h) of the $t_{0}(E)$ curves for different $V_{\mathrm{i}}$ shows that in the nearly free electron region the motion in a nonabsorbing medium is slower than with $V_{\mathrm{i}} \neq 0$, the speed gain being $20 \%$ at $V_{\mathrm{i}}=1.65 \mathrm{eV}$. An obvious reason is that the slower components of the wave packet are stronger damped. In the gap vicinity, however, the advancement is much larger and the dependence on $V_{\mathrm{i}}$ is much stronger. Here we must recall that inelastic scattering is accompanied by the dephasing of the wave packet, which is neglected in the optical potential approach. Thus, we need to establish that the artificial coherence does not alter the result. The escape time $\tau(E)$ obtained with a real potential $\sigma_{n}(t, z)$ (a random function of both time and coordinate) are presented in Fig. 2(g) for emission from the second and fourth layer and in Fig. 2(i) for the first layer (large circles). The stochastic perturbation was chosen such as to give the same MFP as $V_{\mathrm{i}}=1.7 \mathrm{eV}$, Supplemental Material E [17]. The dephasing broadens the $\tau(E)$ features, but otherwise the incoherent ensemble behaves the same as the coherent packet in the 


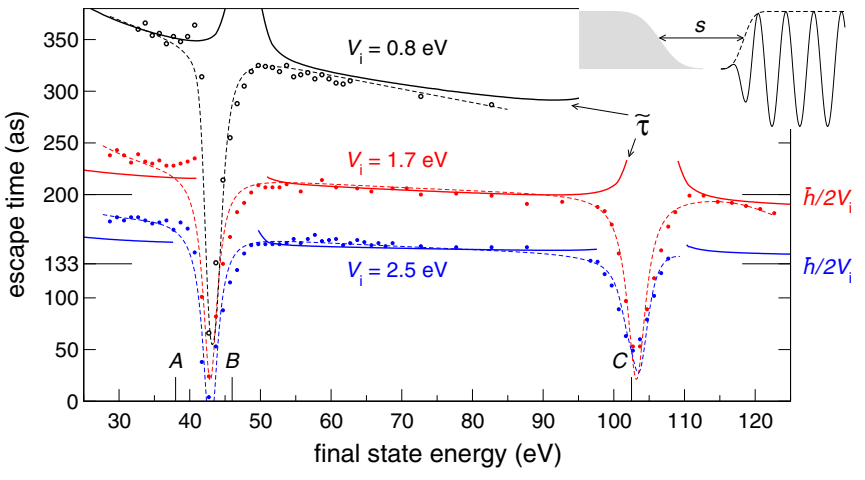

FIG. 4. Escape time as a function of the final state energy for valence band emission. Streaking clock results (circles) are compared with $\tilde{\tau}$ obtained from Eq. (2) (thick curves) with $s=1.75$ a.u. and $w=97.25$ a.u. Three values of the optical potential are considered: $V_{\mathrm{i}}=0.8,1.7$, and $2.5 \mathrm{eV}$. Thin dashed lines are the guide for the eye. Letters $A, B$, and $C$ indicate the intensity minima as shown in Fig. 2(f).

absorbing medium. For the localized states considered above the electron initial position was known. On the contrary, a Bloch state is coherently excited over a depth much larger than MFP, so the initial position depends on MFP. Let us return to the original question of whether Eq. (1) holds in photoemission. We consider the valence band of the crystal of Fig. 2(a) given by a set of discrete states, each extending over the slab of thickness 96 a.u. (MFP is 10-20 a.u.). Escape time $\tau$ is obtained from the streaking curves [as in Figs. 2(c) and 2(d)] for the energy centroid of the sum of the spectra from all the initial states. Figure 4 shows the results for $\hbar \omega=65$ to $160 \mathrm{eV}$ (VB is centered at $-37.3 \mathrm{eV}$, see Supplemental Material C [17]).

Even far from the spectral gaps $\tau(E)$ is not constant but slowly decreases with energy. This, however, does not disprove the idea behind Eq. (1) since this happens because the initial states density edge does not coincide with the onset of the streaking field, see inset of Fig. 4. The generalization of Eq. (1) to the case of a finite distance $s$ between the two planes and a finite slab thickness $w$ reads, see Supplemental Material A [17]:

$$
\tilde{\tau}(v)=\tau+\frac{s}{v}+\frac{w / v}{1-\exp (w / v \tau)} .
$$

Between $E=50$ and $90 \mathrm{eV}$ the curves for three values of $V_{\mathrm{i}}$ are well fitted by Eq. (2) with $s=1.75$ a.u., which agrees with the nominal distance of 2.75 a.u. between the crystal edge and the laser field drop off. These calculations support the initial idea that Bloch electrons can be thought of as starting at the MFP depth $\lambda$ and moving with the group velocity. However, at the energies where the group velocity drops due to the Bragg scattering the escape time becomes shorter and, moreover, the wave packet leaves the crystal earlier than it would have if it moved in a constant (inner) potential.

This result is quite relevant to $\mathrm{Mg}(0001)$, as illustrated in Figs. 1(a) and 1(b): In the experiment of Ref. [9] the emission from the VB overlaps with a continuous fragment of the conducting spectrum, whereas the $\mathrm{Mg} 2 p$ electrons are excited to a narrow band between two gaps, so the photoelectron undergoes strong lattice scattering, Fig. 1(b). This is just the case when the escape time does not obey Eq. (2), and one may even expect the $\mathrm{Mg} 2 p$ to arrive earlier than the VB electron.

This phenomenon should be distinguished from the wellknown Hartman effect, where the tunneling time through a thick barrier turns out to be shorter than the time required by a free particle to travel the same distance [30,31]. In the streaking experiment, owing to the broad spectrum of the pump pulse, the packet consists mainly of propagating waves, and generally the tunneling is negligible, see Fig. 3(a). Still, a behavior similar to the Hartman effect is observed in the broad gap at $E=105 \mathrm{eV}$ as a minimum of the $t_{0}(E)$ curve for $V_{\mathrm{i}}=0$, see Fig. 2(h): the transport by evanescent waves in the middle of the gap is faster than by the propagating waves at the edges of the gap.

It is often asked, where is the spatial starting point of the outgoing photoelectron packet [8,9,12-16,32,33]? A meaning to the notion "starting point" can be ascribed by the streaking experiment or by measuring the motion at later time. If the location of the initial state is taken for the starting point then at certain energies we observe a curious behavior: the electron starting from the outermost layer is overtaken by the one coming from a deeper layer [Figs. 2(h) and 2(i)]. The discovered influence of the exciting light pulse on the wave packet dynamics offers a way to manipulate the electron transport at the nanoscale by tuning the temporal structure of the pulse. Here two aspects are equally important: by changing the frequency $\omega$ one selects the final state range and by changing the pulse duration one further controls its speed.

This work was supported by the Spanish Ministry of Economy and Competitiveness MINECO (Project No. FIS201348286-C2-1-P). E.E.K. acknowledges the Alexander von Humboldt Foundation.
[1] R. Pazourek, S. Nagele, and J. Burgdörfer, Rev. Mod. Phys. 87, 765 (2015).

[2] Z. Tao, C. Chen, T. Szilvási, M. Keller, M. Mavrikakis, H. Kapteyn, and M. Murnane, Science 353, 62 (2016).

[3] F. Krausz and M. Ivanov, Rev. Mod. Phys. 81, 163 (2009).

[4] M. Hentschel, R. Kienberger, C. Spielmann, G. A. Reider, N. Milosevic, T. Brabec, P. Corkum, U. Heinzmann, M. Drescher, and F. Krausz, Nature (London) 414, 509 (2001).
[5] J. Itatani, F. Quéré, G. L. Yudin, M. Y. Ivanov, F. Krausz, and P. B. Corkum, Phys. Rev. Lett. 88, 173903 (2002).

[6] M. Drescher, M. Hentschel, R. Kienberger, G. Tempea, C. Spielmann, G. A. Reider, P. B. Corkum, and F. Krausz, Science 291, 1923 (2001).

[7] R. Kienberger, E. Goulielmakis, M. Uiberacker, A. Baltuska, V. Yakovlev, F. Bammer, A. Scrinzi, T. Westerwalbesloh, 
U. Kleineberg, U. Heinzmann, M. Drescher, and F. Krausz, Nature (London) 427, 817 (2004).

[8] A. L. Cavalieri, N. Muller, T. Uphues, V. S. Yakovlev, A. Baltuska, B. Horvath, B. Schmidt, L. Blumel, R. Holzwarth, S. Hendel, M. Drescher, U. Kleineberg, P. M. Echenique, R. Kienberger, F. Krausz, and U. Heinzmann, Nature (London) 449, 1029 (2007).

[9] S. Neppl, R. Ernstorfer, E. M. Bothschafter, A. L. Cavalieri, D. Menzel, J. V. Barth, F. Krausz, R. Kienberger, and P. Feulner, Phys. Rev. Lett. 109, 087401 (2012).

[10] S. Neppl, R. Ernstorfer, A. L. Cavalieri, C. Lemell, G. Wachter, E. Magerl, E. M. Bothschafter, M. Jobst, M. Hofstetter, U. Kleineberg, J. V. Barth, D. Menzel, J. Burgdorfer, P. Feulner, F. Krausz, and R. Kienberger, Nature (London) 517, 342 (2015).

[11] E. E. Krasovskii, V. M. Silkin, V. U. Nazarov, P. M. Echenique, and E. V. Chulkov, Phys. Rev. B 82, 125102 (2010).

[12] J. C. Baggesen and L. B. Madsen, Phys. Rev. A 78, 032903 (2008).

[13] C. Lemell, B. Solleder, K. Tőkési, and J. Burgdörfer, Phys. Rev. A 79, 062901 (2009).

[14] A. K. Kazansky and P. M. Echenique, Phys. Rev. Lett. 102, 177401 (2009).

[15] A. G. Borisov, D. Sánchez-Portal, A. K. Kazansky, and P. M. Echenique, Phys. Rev. B 87, 121110 (2013).

[16] C. Lemell, S. Neppl, G. Wachter, K. Tőkési, R. Ernstorfer, P. Feulner, R. Kienberger, and J. Burgdörfer, Phys. Rev. B 91, 241101 (2015).

[17] See Supplemental Material at http://link.aps.org/supplemental/ 10.1103/PhysRevB.94.195434 for a derivation of Eqs. (1) and (2) and for details of the methodology.

[18] E. E. Krasovskii, K. Rossnagel, A. Fedorov, W. Schattke, and L. Kipp, Phys. Rev. Lett. 98, 217604 (2007).
[19] E. E. Krasovskii, W. Schattke, P. Jiříček, M. Vondráček, O. V. Krasovska, V. N. Antonov, A. P. Shpak, and I. Bartoš, Phys. Rev. B 78, 165406 (2008).

[20] V. N. Strocov, E. E. Krasovskii, W. Schattke, N. Barrett, H Berger, D. Schrupp, and R. Claessen, Phys. Rev. B 74, 195125 (2006).

[21] E. E. Krasovskii, J. Höcker, J. Falta, and J. I. Flege, J. Phys.: Condens. Matter 27, 035501 (2015).

[22] S. Neppl, Ph.D. thesis, Technische Universität München, 2012.

[23] D. W. Jepsen, F. J. Himpsel, and D. E. Eastman, Phys. Rev. B 26, 4039 (1982).

[24] N. Barrett, E. E. Krasovskii, J.-M. Themlin, and V. N. Strocov, Phys. Rev. B 71, 035427 (2005).

[25] In Fig. 1(b), the group velocity is calculated as the expectation value of the operator $-i \hbar \nabla / m$, and it is always positive, even though in Fig. 1(a) the fragments of the conducting complex band structure that correspond to evanescent waves in the spectral gaps have negative slope.

[26] C. Friedrich, S. Blügel, and A. Schindlmayr, Phys. Rev. B 81, 125102 (2010).

[27] E. E. Krasovskii, Phys. Rev. B 84, 195106 (2011).

[28] E. E. Krasovskii and M. Bonitz, Phys. Rev. Lett. 99, 247601 (2007)

[29] The negative values of $\tau$ do not violate causality because $t=0$ is the center of the XUV pulse, and emission begins at $t=$ -500 asec, see also Supplemental Material F [17].

[30] T. E. Hartman, J. Appl. Phys. 33, 3427 (1962).

[31] H. G. Winful, Phys. Rep. 436, 1 (2006).

[32] C.-H. Zhang and U. Thumm, Phys. Rev. Lett. 102, 123601 (2009).

[33] Q. Liao and U. Thumm, Phys. Rev. A 92, 031401 (2015). 